\title{
A cross-brain regions study of ANK1 DNA methylation in different neurodegenerative diseases
}

DOI:

10.1016/j.neurobiolaging.2018.09.024

\section{Document Version}

Accepted author manuscript

Link to publication record in Manchester Research Explorer

\section{Citation for published version (APA):}

Smith, A. R., Smith, R. G., Burrage, J., Troakes, C., Al-Sarraj, S., Kalaria, R. N., Sloan, C., Robinson, A. C., Mill, J., \& Lunnon, K. (2019). A cross-brain regions study of ANK1 DNA methylation in different neurodegenerative diseases. Neurobiology of Aging, 74, 70-76. https://doi.org/10.1016/j.neurobiolaging.2018.09.024

\section{Published in:}

Neurobiology of Aging

\section{Citing this paper}

Please note that where the full-text provided on Manchester Research Explorer is the Author Accepted Manuscript or Proof version this may differ from the final Published version. If citing, it is advised that you check and use the publisher's definitive version.

\section{General rights}

Copyright and moral rights for the publications made accessible in the Research Explorer are retained by the authors and/or other copyright owners and it is a condition of accessing publications that users recognise and abide by the legal requirements associated with these rights.

\section{Takedown policy}

If you believe that this document breaches copyright please refer to the University of Manchester's Takedown Procedures [http://man.ac.uk/04Y6Bo] or contact uml.scholarlycommunications@manchester.ac.uk providing relevant details, so we can investigate your claim.

\section{OPEN ACCESS}




\section{A cross-brain-regions study of ANK1 DNA methylation in} different neurodegenerative diseases.

9 Adam R Smith ${ }^{1}$, Rebecca G Smith ${ }^{1}$, Joe Burrage ${ }^{1}$, Claire Troakes ${ }^{2}$, Safa Al-Sarraj ${ }^{2}$,

10 Rajesh N Kalaria ${ }^{3}$, Carolyn Sloan ${ }^{4}$, Andrew C Robinson ${ }^{5}$, Jonathan Mill ${ }^{1}$, Katie 11 Lunnon ${ }^{1, *}$

$14{ }^{1}$ University of Exeter Medical School, University of Bristol, Exeter, UK.

152 Institute of Psychiatry, Psychology and Neuroscience (loPPN), King's College 16 London, London, UK.

$17{ }^{3}$ Institute of Neuroscience, Newcastle University, Newcastle, UK.

$18{ }^{4}$ Nuffield Department of Clinical Neurosciences, University of Oxford, Oxford, UK.

$19{ }^{5}$ Faculty of Biology, Medicine and Health, University of Manchester, Manchester, 20 UK.

23 * Corresponding author: Katie Lunnon, University of Exeter Medical School, RILD 24 Building Level 4, Royal Devon and Exeter Hospital, Barrack Rd, Exeter. EX2 5DW. 25 UK. E-mail: k.lunnon@exeter.ac.uk license http://creativecommons.org/licenses/by-nc-nd/4.0/ 
3 Recent epigenome-wide association studies in Alzheimer's disease have highlighted

4 consistent robust neuropathology-associated DNA hypermethylation of the Ankyrin 1 5 (ANK1) gene in the cortex. The extent to which altered ANK1 DNA methylation is 6 also associated with other neurodegenerative diseases is not currently known. In the 7 current study, we used bisulfite pyrosequencing to quantify DNA methylation across 8 eight $\mathrm{CpG}$ sites within a 118bp region of the ANK1 gene across multiple brain 9 regions in Alzheimer's disease, Vascular dementia, Dementia with Lewy bodies, 10 Huntington's disease and Parkinson's disease. We demonstrate disease-associated 11 ANK1 hypermethylation in the entorhinal cortex in Alzheimer's disease, Huntington's 12 disease and Parkinson's disease, whilst in donors with Vascular dementia and 13 Dementia with Lewy bodies we observed elevated ANK1 DNA methylation only in 14 individuals with co-existing Alzheimer's disease pathology. We did not observe any 15 disease-associated differential ANK1 DNA methylation in the striatum in Huntington's 16 disease, or the substantia nigra in Parkinson's disease. Our data suggests that ANK1 17 is characterized by region and disease-specific differential DNA methylation in 18 multiple neurodegenerative diseases.

\section{Keywords:}

22 Alzheimer's disease (AD); Ankyrin 1 (ANK1); Brain; Dementia with Lewy Bodies 23 (DLB); DNA methylation (5-methylcytosine - 5mC); Epigenetics; Huntington's 24 disease (HD); Parkinson's disease (PD); Vascular dementia (VaD) 
3 Dementia encompasses a group of chronic neurodegenerative diseases that affected

4 an estimated 46.8 million people worldwide in 2015 (Wimo et al., 2017), of which

5 Alzheimer's disease (AD) accounts for $\sim 60 \%$ of cases. The etiology of AD has been

6 hypothesized to involve epigenetic mechanisms (Lunnon and Mill, 2013). In 2014 two

7 epigenome-wide association studies (EWAS) of AD identified significant

8 hypermethylation of $\mathrm{CpG}$ sites in the Ankyrin 1 (ANK1) gene associated with

9 neuropathology in AD cortex (De Jager et al., 2014; Lunnon et al., 2014), which has

10 been replicated in multiple independent study cohorts (Smith, 2017). Subsequently, a

11 genome wide association study (GWAS) of a Han Chinese population identified a

12 single-nucleotide polymorphism (SNP) in ANK1 associated with an increased

13 susceptibility for developing AD (Chi et al., 2015). ANK1 links integral membrane

14 proteins to the underlying spectrin-actin cytoskeleton and plays a key role in cell

15 motility, activation, proliferation, contact, and maintenance of specialized membrane

16 domains (Yang et al., 2011). There is now increasing interest in understanding the

17 role of epigenetic changes in ANK1 in the development and progression of AD. One

18 important question to be addressed is whether ANK1 hypermethylation is specific to

$19 A D$, or observed in other neurodegenerative disorders. Although $A D$ accounts for

$20 \sim 60 \%$ of dementia cases, many other dementias share common symptoms and/or

21 pathological hallmarks with AD.

23 This study aimed to quantify DNA methylation levels across a $118 \mathrm{bp}$ region of $A N K 1$,

24 previously associated with $A D$, in a number of different neurodegenerative diseases.

25 Using bisulfite pyrosequencing we assessed ANK1 DNA methylation in brain

26 samples from donors with AD, Dementia with Lewy bodies (DLB), Vascular dementia

$27(\mathrm{VaD})$, Huntington's disease (HD), Parkinson's disease (PD) and non-demented 
1 elderly controls, across a number of different brain regions that are characterized by

2 disease-specific pathology.

\section{MATERIALS AND METHODS}

Post-mortem brain tissue was obtained from six different UK brain banks (the South

9 West Dementia Brain Bank (SWDBB), the London Neurodegenerative Disease Brain 10 Bank (LNDBB) the Manchester Brain Bank, the Oxford Brain Bank, the Cambridge

11 Brain Bank and the Newcastle Brain Bank). In total, tissue was obtained from $60 \mathrm{AD}$ 12 (Braak V-VI), 119 DLB, 27 VaD, 22 HD, 36 PD and 105 elderly non-demented control subjects (Braak 0-II). A subset of DLB $(\mathrm{N}=39)$ and $\operatorname{VaD}(\mathrm{N}=5)$ cases also had co-

14 existing $A D$ pathology. For each disease we analyzed the entorhinal cortex (EC), 15 superior temporal gyrus (STG) and cerebellum (CER). For HD cases we also 16 analyzed the striatum (STR) as this is primarily affected in disease (Reiner et al., 17 2011), whilst for PD cases we analyzed the STR and substantia nigra (SN) as these 18 are regions of pathology in this disease (Fearnley and Lees, 1991). For control 19 samples we analyzed all five brain regions. For a small number of donors tissue was not available from all brain regions. Genomic DNA was isolated from $100 \mathrm{mg}$ of each

21 dissected brain region using a standard phenol-chloroform extraction method, and 22 tested for degradation and purity prior to analysis as previously described (Smith et 23 al., 2016). Demographic information for samples can be found in Supplementary 24 Table 1.

\subsection{ANK1 bisulfite pyrosequencing}

27 Bisulfite pyrosequencing was used to quantify DNA methylation across eight 28 individual CpG sites in the ANK1 gene, spanning from 41519302 to 41519420 within 
1 chromosome 8 (hg19). Bisulfite conversion was performed using the Bisulfite-Gold kit

2 (Zymo research, USA). A single amplicon (246bp) was generated using primers

3 designed using the PyroMark Assay Design software 2.0 (Qiagen, UK) as previously

4 described (Lunnon et al., 2014). Pyrosequencing was performed using two

5 sequencing primers to maximize coverage across eight $\mathrm{CpG}$ sites. DNA methylation

6 was quantified using the Pyromark Q24 system (Qiagen, UK) following the

7 manufacturer's standard instructions and the Pyro Q24 CpG 2.0.6 software.

8

\subsection{Data Analysis}

All computations and statistical analyses were performed using $R$ 3.3.2 ( $R$

11 Development Core Team, 2012). A linear regression analysis was performed, 12 controlling for the effects of age, gender and batch effects, comparing control 13 samples with samples affected by each neurodegenerative disease. For the VaD and 14 DLB samples we also performed a second analysis to investigate whether co-existing 15 AD pathology influenced the results by comparing individuals with and without co16 existing $A D$ pathology to control samples, again accounting for the effects of age, 17 gender and batch. Our analyses examined DNA methylation differences at (a) 18 individual $\mathrm{CpG}$ sites and (b) averaged across the amplicon. We used a paired two19 tailed t-test to compare adjusted DNA methylation differences in disease across brain 20 regions.

\section{RESULTS}

\subsection{AD-associated ANK1 DNA hypermethylation is seen across all tissues} analyzed

27 First, we sought to replicate previous findings of ANK1 DNA hypermethylation in AD.

28 Across the 118bp region, we observed significantly increased levels in AD cases 
1 compared to controls in all eight ANK1 CpG sites in the EC (Figure 1A) and seven

2 ANK1 CpG sites in the STG (Figure 1B). Both the EC and STG exhibit a high degree

3 of $A D$ pathology, even in the earliest stages of disease, with the $E C$ being the starting

4 point of $A D$ pathology in the cortex with pathology seen here in Braak stage II (Braak

5 and Braak, 1991). Conversely, the CER remains free of AD pathology until the very

6 last stages of the disease, although even then this is limited to amyloid beta (A $\beta)$

7 plaques with an absence of neurofibrillary tangles (NFTs) of hyperphosphorylated tau

8 (Braak et al., 1989). Of note, we observed significant ANK1 DNA hypermethylation at

9 five ANK1 CpG sites in the CER (Figure 1C). This is the first time ANK1 DNA 10 methylation changes have been reported in the CER. Interestingly, two of the loci 11 that did not display AD-associated ANK1 hypermethylation in the CER were 12 chr8:41519308 and chr8:41519399, the two sites included on the Illumina 450K array 13 used in previous EWAS analyses of AD which did not identify ANK1 14 hypermethylation in AD in the CER (Lunnon et al., 2014). Average DNA methylation 15 across the amplicon region was significantly elevated in AD in the $\mathrm{EC}\left(P=1.29 \times 10^{-}\right.$ $\left.16^{07}\right)$, STG $\left(P=2.39 \times 10^{-03}\right)$ and CER $\left(P=7.81 \times 10^{-03}\right)$ (Figure 1D). ANK1 DNA 17 methylation differences between cases and controls at both individual sites and 18 across the amplicon were lower in the CER compared to other tissues tested 19 (Supplementary Table 2), with a significantly greater DNA methylation difference 20 between cases and controls in the EC (amplicon average corrected DNA methylation

21 difference $(\Delta)=4.53 \%$ ) compared to both the STG (amplicon average $\Delta=2.84 \% ; P$ $22=7.98 \times 10^{-4}$ ) and the CER (amplicon average $\Delta=1.17 \%, P=2.55 \times 10^{-4}$ ). 23 Interestingly, this pattern of change matches the spread of AD pathology throughout 24 the brain. 
1 DLB is the third most common cause of dementia with the age of onset ranging from

250 to 83 years (McKeith, 2002). The pathology of DLB shares similarities to AD, with

3 the presence of immune regulation and microglial activation being consistent

4 between diseases (Mackenzie, 2000). However, the presence of Lewy bodies within

5 the brain makes DLB considerably more comparable to PD (McKeith, 2002). In fact,

6 PD dementia is thought to be biologically identical to DLB, only differing in the order

7 in which the motor or cognitive symptoms occur (Dodel et al., 2008). Interestingly we

8 observed significant hypermethylation of $A N K 1$ in DLB cases compared to controls in

9 the EC (Figure 2A) at four of the eight ANK1 CpG sites (Supplementary Table 3).

10 We saw no difference between DLB and control samples in either the STG

11 (Supplementary Figure 1A) or the CER (Supplementary Figure 1B) at any of the

12 eight ANK1 CpG sites. Across the ANK1 amplicon we observed significant DLB-

13 associated hypermethylation in the EC $(P=0.0244)$, but not in the STG or CER $(P>$

14 0.05) (Figure 2B). It is widely reported that DLB and AD frequently co-occur

15 (Rosenberg et al., 2001); we were therefore interested to investigate whether we still

16 observed DLB-associated DNA hypermethylation in the EC when we controlled for

17 co-existing AD pathology. We found no significant changes in ANK1 DNA 18 methylation in individuals with "pure" DLB compared to controls in the EC (Figure

19 2C), STG (Supplementary Figure 1C) and CER (Supplementary Figure 1D).

20 However, we did observe significant hypermethylation in DLB cases with co-existing

$21 \mathrm{AD}$ pathology compared to controls at seven of the eight $A N K 1 \mathrm{CpG}$ sites in the EC

22 (Figure 2C) and two sites in the STG (Supplementary Figure 1C), with no 23 difference in the CER (Supplementary Figure 1D). When we looked across the 24 whole 118bp region, we saw increased ANK1 DNA methylation in the EC in 25 individuals with co-existing $\mathrm{AD}$ pathology $\left(P=1.45 \times 10^{-03}\right)$ (Figure 2D), suggesting 26 that the ANK1 hypermethylation seen in some individuals with DLB is primarily driven 27 by AD pathology. 

co-existing AD pathology

3 Characterized by the loss of neurological function due to ischemic events, the risk of 4 developing $\mathrm{VaD}$ is closely linked to vascular health (Román et al., 1993). We observed increased DNA methylation in individuals with $\mathrm{VaD}$ at none of the ANK1 CpG sites in the EC (Figure 3A), only one site in the STG (Supplementary Figure 2A) and no sites in the CER (Supplementary Figure 2B) (Supplementary Table 4), with no difference across the amplicon in any of the brain regions tested $(P>0.05)$ (Figure 3B). Because $\mathrm{VaD}$ also often co-occurs with $\mathrm{AD}$ we next examined whether stratifying cases by the presence of $A D$ pathology altered these findings.

11 Interestingly, we saw disease-associated hypermethylation in the EC at five of the eight ANK1 CpG sites only in individuals with co-existing AD pathology (Figure 3C),

13 whilst we saw disease-associated hypomethylation at one site in the STG

14 (Supplementary Figure 2C) in individuals with "pure" $\mathrm{VaD}$, and no disease15 associated changes in the CER (Supplementary Figure 2D). When we looked 16 across the $118 \mathrm{bp}$ region we only saw significant ANK1 hypermethylation in 17 individuals with $\mathrm{VaD}$ and co-existing $\mathrm{AD}$ pathology compared to controls in the $\mathrm{EC}(P$ $18=0.0163$ ) (Figure 3D). It is worth noting that our cohort only had a small number of $19 \operatorname{VaD}$ cases with co-existing $A D$ pathology $(\mathrm{N}=5)$.

\subsection{ANK1 DNA hypermethylation in the EC is seen in both HD and PD.}

$\mathrm{HD}$ is characterized by a trinucleotide repeat in the huntingtin gene $(H T T)$. The abundance of the repeat is proportional to the level of protein misfolding and downstream cytosolic accumulation, leading to neuronal cell death and the symptoms of HD (Walker, 2007). ANK1 DNA hypermethylation was seen at four of

26 the eight $\mathrm{CpG}$ sites in the EC in HD (Figure 4A). However, no differential DNA 27 methylation was seen in the other brain regions tested (Supplementary Table 5), 28 including the STG (Supplementary Figure 3A), the CER (Supplementary Figure 
1 3B) and the STR (Supplementary Figure 3C), a region that forms part of the basal

2 ganglia, known to be the first brain region to be adversely affected by HD pathology

3 (Walker, 2007). Averaging across the region again highlighted significant

4 hypermethylation in the EC $\left(P=6.68 \times 10^{-3}\right)$, with no significant change in any other

5 tissue (Figure 4B).

7 A similar pattern of $A N K 1$ hypermethylation was observed in PD; two of the eight

$8 \mathrm{CpG}$ sites were characterized by significant hypermethylation in the EC (Figure 4C),

9 with no differences in DNA methylation in any of the other brain regions tested

10 (Supplementary Table 6). This included the STG (Supplementary Figure 4A), the

11 CER (Supplementary Figure 4B), the STR (Supplementary Figure 4C) and the SN

12 (Supplementary Figure 4D), with the SN representing the brain region that has the

13 highest levels of pathology in PD (Fearnley and Lees, 1991). Across the 118bp

14 amplicon we saw no change in DNA methylation in any of the five brain regions 15 (Figure 4D).

\section{DISCUSSION}

This is the first study to assess brain ANK1 DNA methylation changes across

21 multiple neurodegenerative diseases. We identified significant DNA methylation 22 changes in the EC in multiple diseases, including $A D, H D$ and $P D$, with significant 23 DNA hypermethylation across the amplicon in AD and HD. Interestingly, we also 24 observed significant hypermethylation of $A N K 1$ in the EC in both DLB and VaD at 25 several individual $\mathrm{CpG}$ sites and across the amplicon, but only in donors with co26 existing $\mathrm{AD}$ pathology. This suggests that $A N K 1$ DNA hypermethylation in the $\mathrm{EC}$ is 27 specific to some neurodegenerative diseases (AD, HD and to some extent PD), and 28 not observed in other forms of neuropathology ( $\mathrm{VaD}$ and $\mathrm{DLB})$. Although it is possible 
1 that the observed changes in ANK1 could reflect a common feature of

2 neurodegenerative diseases, such as neuroinflammation, it is worth noting that we

3 did not observe ANK1 DNA hypermethylation in all diseases, for example we did not

4 see any hypermethylation in individuals with "pure" DLB or VaD. These diseases are

5 also characterised by neuroinflammation, so this suggests that the observed

6 hypermethylation does not simply reflect a common hallmark of all

7 neurodegenerative diseases such as microgliosis. We have previously reported that

8 ANK1 is not hypermethylated in the CER in AD at two sites interrogated by the

9 Illumina 450K array (chr8:41519308 and chr8:41519399). In the current study we

10 again demonstrate that these two loci are not significantly differentially methylated in

11 AD; however, we do highlight AD-associated DNA hypermethylation at five adjacent

12 CpG sites and averaged across the 118bp amplicon in the CER. We did not see any

13 ANK1 DNA methylation changes in the CER in any of the other neurodegenerative

14 diseases, including those with co-existing AD pathology. Reflecting our previous

15 findings, we found that DNA methylation differences in $A D$ are greatest in the $E C$, an

16 area with high levels of neuropathology and lowest in the CER, the region with the

17 least neuropathology. Interestingly, although we observed disease-associated ANK1

18 hypermethylation in the EC at six individual sites and across the region in HD and at

19 two individual sites in PD, we did not see ANK1 DNA methylation changes in these

20 diseases in their regions of primary neuropathology, namely the STR and SN,

21 respectively. This suggests that $A N K 1$ hypermethylation in neurodegenerative

22 disease is not necessarily specific to regions of primary neuropathology, but may

23 instead be specific to particular cell types affected in only specific diseases, such as

24 those in the EC, which are not present in the STR and SN. ANK1 encodes for

25 numerous isoforms with their own tissue-specific enhancers. Although the precise

26 function of most $A N K 1$ isoforms is not known, different isoforms have been identified

27 in the brain, blood and muscle (Gallagher et al., 1997). It would be of interest to

28 examine expression levels of different $A N K 1$ transcript variants, to facilitate the 
1 interpretation of the DNA methylation differences we observe. Mastroeni et al.,

2 recently showed a four-fold increase in ANK1 mRNA expression in microglia from AD

3 brain tissue, but not in neurons or astrocytes from the same individuals, suggesting

4 an immune based function for ANK1 in the human brain (Mastroeni et al., 2017). One

5 potential caveat of our study is that we have analyzed "bulk" tissue, and we cannot

6 determine which cell type(s) are driving the DNA hypermethylation seen in ANK1 in 7 disease.

9 Although the focus of our study was on investigating DNA methylation changes in 10 disease, bisulfite pyrosequencing actually generates a summative measurement of 11 both DNA methylation and DNA hydroxymethylation. DNA methylation is generally 12 associated with gene silencing, particularly when residing in the promoters of 13 constitutively expressed housekeeping genes (Jones, 2012), whilst DNA 14 hydroxymethylation has been shown to be enriched in gene bodies (Lunnon et al., 15 2016) and to be found at (relatively) high levels in the brain (Khare et al., 2012; 16 Szulwach et al., 2011). We have recently shown that ANK1 DNA hypermethylation 17 across the $118 \mathrm{bp}$ amplicon in $A D$ is actually underestimated when using bisulfite 18 data, as it is confounded by significant DNA hypohydroxymethylation at some loci in 19 the amplicon (Smith et al., Under Review). Another caveat to our study is that we 20 have only analyzed DNA methylation across eight CpG sites in a 118bp region of the 21 ANK1 gene and thus future studies should aim to further quantify changes in DNA 22 methylation across the entire $244 \mathrm{~kb}$ gene.

\section{CONCLUSIONS}

26 Our study has demonstrated disease-associated $A N K 1$ hypermethylation in the EC at 27 specific $\mathrm{CpG}$ sites in $\mathrm{AD}, \mathrm{HD}$ and $\mathrm{PD}$ and across the region in $\mathrm{AD}$ and $\mathrm{HD}$. In donors 28 with DLB and $\mathrm{VaD}$ we only observed increased ANK1 DNA methylation in the EC in 
1 individuals with co-existing AD pathology. The CER showed disease-associated

2 hypermethylation at specific $\mathrm{CpG}$ loci and across the region in $\mathrm{AD}$, but not in any of

3 the other neurodegenerative diseases tested. We saw no disease-associated

4 differential ANK1 DNA methylation in the STR in HD or PD, or the SN in PD. This

5 suggests that $A N K 1$ is characterized by brain region and disease-specific differential

6 DNA methylation in different neurodegenerative diseases. It is unlikely that the

7 identified DNA methylation changes in $A N K 1$ could be useful as a biomarker

8 clinically, as we have previously shown that $A N K 1$ is not hypermethylated in blood in

$9 \mathrm{AD}$ (Lunnon et al., 2014). However, these epigenetic changes could represent novel

10 therapeutic targets for disease, if shown to be causal in pathology. At present we are

11 unable to determine whether these changes represent a cause or a consequence of

12 the disease process. Further functional studies should therefore be performed to

13 determine the potential disease causality of this modification.

14

15

\section{ACKNOWLEDGEMENTS}

This work was funded by an Alzheimer's Association US New Investigator Research Grant (NIRG-14-320878) to KL, a grant from BRACE (Bristol Research into Alzheimer's and Care of the Elderly) to KL, a major project grant from the Alzheimer's Society UK (AS-PG-14-038) to KL and an Equipment Grant from Alzheimer's Research UK (ART-EG2010A-2) to JM. We thank all the donors and families who have made this research possible. Brain tissue was received from six of the UK Brain Banks. Brain tissue collection by the Newcastle brain bank, Oxford Brain Bank, The London Neurodegenerative Diseases Brain Bank (LNDBB) and the South West Dementia Brain Bank (SWDBB) is supported by the UK Medical Research Council (G0400074). The Newcastle Brain Bank, Oxford Brain Bank, LNDBB, SWDBB and

\footnotetext{
Manchester Brain Bank are all supported by the Brains for Dementia Research
} 
(BDR) program, jointly funded by Alzheimer's Research UK and Alzheimer's Society.

In addition, the Newcastle Brain Bank is also supported by the NIHR Newcastle

Biomedical Research Centre and Unit award to the Newcastle upon Tyne NHS

Foundation Trust and Newcastle University. The Oxford Brain Bank is also supported by Autistica UK and the NIHR Oxford Biomedical Research Centre. The SWDBB is also supported by BRACE. The Cambridge Brain Bank is supported by the NIHR and the Cambridge Biomedical Research Centre.

\section{REFERENCES}

Braak, H., Braak, E., 1991. Neuropathological stageing of Alzheimer-related changes. Acta neuropathologica 82(4), 239-259.

Braak, H., Braak, E., Bohl, J., Lang, W., 1989. Alzheimer's disease: amyloid plaques in the cerebellum. Journal of the Neurological Sciences 93(2), 277-287.

Chi, S., Song, J.H., Tan, M.S., Zhang, W., Wang, Z.X., Jiang, T., Tan, L., Yu, J.T., 2015. Association of Single-Nucleotide Polymorphism in ANK1 with Late-Onset Alzheimer's Disease in Han Chinese. Mol Neurobiol.

De Jager, P.L., Srivastava, G., Lunnon, K., Burgess, J., Schalkwyk, L.C., Yu, L., Eaton, M.L., Keenan, B.T., Ernst, J., McCabe, C., Tang, A., Raj, T., Replogle, J., Brodeur, W., Gabriel, S., Chai, H.S., Younkin, C., Younkin, S.G., Zou, F., Szyf, M., Epstein, C.B., Schneider, J.A., Bernstein, B.E., Meissner, A., Ertekin-Taner, N., Chibnik, L.B., Kellis, M., Mill, J., Bennett, D.A., 2014. Alzheimer's disease: early alterations in brain DNA methylation at ANK1, BIN1, RHBDF2 and other loci. Nat Neurosci 17(9), 1156-1163.

Dodel, R., Csoti, I., Ebersbach, G., Fuchs, G., Hahne, M., Kuhn, W., Oechsner, M., Jost, W., Reichmann, H., Schulz, J.B., 2008. Lewy body dementia and Parkinson's disease with dementia. Journal of neurology 255 Suppl 5, 39-47.

Fearnley, J.M., Lees, A.J., 1991. Aging and Parkinson's disease: substantia nigra regional selectivity. Brain : a journal of neurology 114(5), 2283-2301. Gallagher, P.G., Tse, W.T., Scarpa, A.L., Lux, S.E., Forget, B.G., 1997. Structure and Organization of the Human Ankyrin-1 Gene: BASIS FOR COMPLEXITY OF PREmRNA PROCESSING. Journal of Biological Chemistry 272(31), 19220-19228. Jones, P.A., 2012. Functions of DNA methylation: islands, start sites, gene bodies and beyond. Nature reviews. Genetics 13.

Khare, T., Pai, S., Koncevicius, K., Pal, M., Kriukiene, E., Liutkeviciute, Z., Irimia, M., Jia, P., Ptak, C., Xia, M., Tice, R., Tochigi, M., Morera, S., Nazarians, A., Belsham, D., Wong, A.H., Blencowe, B.J., Wang, S.C., Kapranov, P., Kustra, R., Labrie, V., Klimasauskas, S., Petronis, A., 2012. 5-hmC in the brain is abundant in synaptic genes and shows differences at the exon-intron boundary. Nature structural \& molecular biology 19(10), 1037-1043.

Lunnon, K., Hannon, E., Smith, R.G., Dempster, E., Wong, C., Burrage, J., Troakes, C., Al-Sarraj, S., Kepa, A., Schalkwyk, L., Mill, J., 2016. Variation in 5- 
hydroxymethylcytosine across human cortex and cerebellum. Genome biology $17,27$.

Lunnon, K., Mill, J., 2013. Epigenetic studies in Alzheimer's disease: current findings, caveats, and considerations for future studies. Am J Med Genet B Neuropsychiatr Genet 162B(8), 789-799.

Lunnon, K., Smith, R., Hannon, E., De Jager, P.L., Srivastava, G., Volta, M., Troakes, C., Al-Sarraj, S., Burrage, J., Macdonald, R., Condliffe, D., Harries, L.W., Katsel, P., Haroutunian, V., Kaminsky, Z., Joachim, C., Powell, J., Lovestone, S., Bennett, D.A., Schalkwyk, L.C., Mill, J., 2014. Methylomic profiling implicates cortical deregulation of ANK1 in Alzheimer's disease. Nature neuroscience 17(9), 11641170.

Mackenzie, I.R.A., 2000. Activated microglia in dementia with Lewy bodies. Neurology 55(1), 132-134.

Mastroeni, D., Sekar, S., Nolz, J., Delvaux, E., Lunnon, K., Mill, J., Liang, W.S., Coleman, P.D., 2017. ANK1 is up-regulated in laser captured microglia in Alzheimer's brain; the importance of addressing cellular heterogeneity. PloS one 12(7), e0177814.

McKeith, I.G., 2002. Dementia with Lewy bodies. The British Journal of Psychiatry 180(2), 144-147.

R Development Core Team, 2012. R: A Language and Environment for Statistical Computing. R Foundation for Statistical Computing, Vienna, Austria 2012.

Reiner, A., Dragatsis, I., Dietrich, P., 2011. GENETICS AND NEUROPATHOLOGY OF HUNTINGTON'S DISEASE. International review of neurobiology 98, 325-372. Román, G.C., Tatemichi, T.K., Erkinjuntti, T., Cummings, J.L., Masdeu, J.C., Garcia, J.H., Amaducci, L., Orgogozo, J.-M., Brun, A., Hofman, A., Moody, D.M., O'Brien, M.D., Yamaguchi, T., Grafman, J., Drayer, B.P., Bennett, D.A., Fisher, M., Ogata, J., Kokmen, E., Bermejo, F., Wolf, P.A., Gorelick, P.B., Bick, K.L., Pajeau, A.K., Bell, M.A., DeCarli, C., Culebras, A., Korczyn, A.D., Bogousslavsky, J., Hartmann, A., Scheinberg, P., 1993. Vascular dementia. Diagnostic criteria for research studies: Report of the NINDS - AIREN International Workshop* 43(2), 250-250. Rosenberg, C.K., Cummings, T.J., Saunders, A.M., Widico, C., McIntyre, L.M., Hulette, C.M., 2001. Dementia with Lewy bodies and Alzheimer's disease. Acta Neuropathol 102(6), 621-626.

Smith, A.R., Smith, R.G., Condliffe, D., Hannon, E., Schalkwyk, L., Mill, J., Lunnon, K., 2016. Increased DNA methylation near TREM2 is consistently seen in the superior temporal gyrus in Alzheimer's disease brain. Neurobiology of aging 47, 35-40.

Smith, A.R., Smith, R.G., Hannon, E., Roubroeks, J.A.Y., Burrage, J., Troakes, C., AlSarraj, S., Mill, J., van den Hove, D.L., Lunnon, K., Under Review. Parallel profiling of DNA methylation and hydroxymethylation highlights neuropathologyassociated epigenetic variation within ANK1 and thirteen novel loci in Alzheimer's disease entorhinal cortex. Genome biology.

Smith, R.G.L., K., 2017. DNA Modifiactions and Alzheimer's Disease, in: DelgadoMorales, R. (Ed.) Neuroepigenomics in Aging and Disease. . Springer International Publishing, pp. 303-319.

Szulwach, K.E., Li, X., Li, Y., Song, C.X., Wu, H., Dai, Q., Irier, H., Upadhyay, A.K., Gearing, M., Levey, A.I., Vasanthakumar, A., Godley, L.A., Chang, Q., Cheng, X., He, C., Jin, P., 2011. 5-hmC-mediated epigenetic dynamics during postnatal neurodevelopment and aging. Nature neuroscience 14(12), 1607-1616. 
1 Walker, F.O., 2007. Huntington's disease. The Lancet 369(9557), 218-228.

2 Wimo, A., Guerchet, M., Ali, G.C., Wu, Y.T., Prina, A.M., Winblad, B., Jonsson, L., Liu,

3 Z., Prince, M., 2017. The worldwide costs of dementia 2015 and comparisons

4 with 2010. Alzheimer's \& dementia : the journal of the Alzheimer's Association

5 13(1), 1-7.

6 Yang, M., Ge, W., Chowdhury, R., Claridge, T.D., Kramer, H.B., Schmierer, B.,

7 McDonough, M.A., Gong, L., Kessler, B.M., Ratcliffe, P.J., Coleman, M.L., Schofield,

8 C.J., 2011. Asparagine and aspartate hydroxylation of the cytoskeletal ankyrin

9 family is catalyzed by factor-inhibiting hypoxia-inducible factor. The Journal of

10 biological chemistry 286(9), 7648-7660. 